\title{
MODELO DE PLANEACIÓN DE RUTAS PARA LOS USUARIOS DE BOGOTÁ
}

\author{
ROUTE PLANNING MODEL FOR USERS IN BOGOTA \\ Wilson Vargas ${ }^{a, b, c,{ }^{*},}$, Carmen Femenia ${ }^{b}$ \\ a Universidad Distrital Francisco José de Caldas, Bogotá, Distrito Capital, Colombia.wvargas@udistrital.edu.co

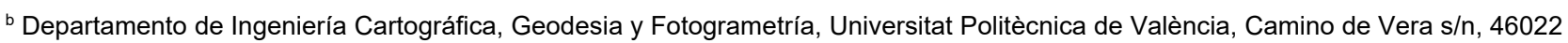 \\ Valencia, España.wevarvar@upv.edu.es; cfemenia@cgf.upv.es \\ c Universidad Nacional de Colombia, Bogotá, Distrito Capital, Colombia. wevargas@unal.edu.co
}

\begin{abstract}
:
Bogotá, the capital of Colombia, is the first most congested city in the world after Bucharest and New York. In Bogotá there are several applications (Apps) that allow the user to plan their trip in each of the different existing modes of transport, which are based on maps and georeferenced data of the cartographic part as well as traffic variables such as congestion, walking times and mode to use for the trip. In recent years for users the variable that determines their planning is not travel time, but additional factors that applications do not take into account such as: occupation of means of transport, stops and portals, infrastructure operation, environmental conditions, citizen security among others. The objective of the research work is to establish a model that allows the different road actors to plan the routes according to their needs and based on the real travel time. The needs of the users will be determined by means of field surveys to obtain the additional travel times of each of the variables found. The project intends to improve travel planning for each of the road users (drivers, passengers, pedestrians and bi-users) that allow for more real travel times, that allow improving the mobility of a city as congested as Bogotá.
\end{abstract}

Key words: mobility, route planning, app, cartography

\section{Resumen:}

Bogotá, la capital de Colombia, es la primera ciudad más congestionada del mundo después de Bucarest y Nueva York. En Bogotá existen varias aplicaciones (Apps) que le permiten al usuario planear su viaje en cada uno de los diferentes modos de transporte existentes, las cuales se basan en mapas y datos georreferenciados de la parte cartográfica como de variables de transito como congestión, tiempos de caminata y modo a utilizar para el viaje. En los últimos años para los usuarios la variable que determina su planeación no es el tiempo de viaje, sino factores adicionales que las aplicaciones no tienen en cuenta como: ocupación de los medios de transporte, paraderos y portales, operación de la infraestructura, condiciones ambientales, seguridad ciudadana entre otros. El objetivo del trabajo de investigación es establecer un modelo que permita a los diferentes actores viales realizar la planeación de las rutas de acuerdo a sus necesidades y en función del tiempo real de viaje. Las necesidades de los usuarios se determinarán por medio de encuestas en campo para obtener los tiempos adicionales de viaje de cada una de las variables encontradas. Con el proyecto se pretende mejorar la planeación de los viajes de cada uno de los usuarios viales (conductores, pasajeros, peatones y biciusuarios) que permita tener tiempos de viaje más reales, que permita mejorar la movilidad de una ciudad tan congestionada como es Bogotá.

Palabras clave: movilidad, planeación rutas, app, cartografía

\section{Antededentes}

Bogotá, la capital colombiana, no es ajena al problema de movilidad, ya que según la consultora Inrix (2020) en un estudio realizado en más de 200 ciudades en 38 países, es la primera ciudad más congestionada del mundo después de Bucarest y Nueva York. Según Carlos César Parrado, en la Columna del Tiempo, plantea que existen seis claves fundamentales para mejorar la movilidad en Bogotá: Mejorar el uso del suelo reduciendo la necesidad de desplazamiento de las personas, Mejorar la coordinación e infraestructura del transporte público y los diferentes estamentos encargados del tema, Continuar la mejora de la infraestructura actual que se dispone para los peatones y los usuarios de la bicicleta, prioridad para el transporte público y el desarrollo de la intermodalidad, continuación del mejoramiento técnico de los vehículos y sus combustibles y Uso racional del carro privado (Parrado 2001).

Un estudio realizado por INRIX Global Traffic Scorecard de 2020 indicó que los bogotanos pierden 133 horas al año, más de 11 días en congestiones (atascos) y embotellamientos. Este factor afecta directamente la salud mental de los ciudadanos y su principal riesgo es el estrés, que según el Observatorio Europeo de Riesgos

`Corresponding Author: Wilson Vargas, wvargas@udistrital.edu.co 
equivale a entre el $50 \%$ y $60 \%$ de los días de trabajo perdidos por una persona. (Quessep-Alcové 2019).

Por lo anterior el futuro de la planeación según el parlamento europeo (ISER 2010) es lograr que los sistemas de transporte tengan un enfoque específico a la hora de abordar la movilidad social que les permita adaptarse a circunstancias y estilos de vida, así como introducir las opciones de transporte adecuadas para el consumidor.

La movilidad durante y después del confinamiento generado en el mundo por el COVID-19, ha cambiado, ya que los objetivos y necesidad de movilidad de los actores viales cambiaron, según el documento movilidad y bienestar durante el confinamiento por covid-19 en la comunitat valenciana (Ruiz-Sánchez et al. 2020), los encuestados que declaran desplazarse durante el confinamiento lo hacen mayoritariamente a pie (59.8\%). En segundo lugar, en orden de importancia se sitúa el uso del coche $(33,8 \%)$. El resto de modos de transporte son utilizados en mucha menor medida: transporte público colectivo $(2,7 \%)$, bicicleta $(1,5 \%)$, Moto $(1,0 \%)$ y otros $(1,1 \%)$.

De igual manera en Colombia, la Universidad Nacional realizó el Primer Encuentro Virtual de Secretarios y Autoridades de Territorio y Movilidad, en el cual una de las primeras conclusiones fue que las necesidades y hábitos de viaje de las personas antes y después de la cuarentena han hecho que la movilidad se perciba desde otro punto de vista, la reinvención y la implementación de nuevas alternativas de transporte sostenible, al igual se planteó que dentro de los cambios a los que van a apostar la mayoría de ciudades, está la implementación de la movilidad sostenible, que se refiere a desplazamiento tanto en bicicletas, patinetas eléctricas y caminatas.

Como un apoyo para mejorar esta movilidad, se ha planteado la utilización de la tecnología y el avance de la misma, en el desarrollo de aplicaciones para teléfonos inteligentes (Apps), con las cuales ayudan a conductores, pasajeros, bici usuarios y peatones a poder planear sus viajes en los diferentes sistemas de transporte colectivo e individual que tiene cada una de las ciudades del mundo, muchos autores como (Sabino et al. 2020) presentan en sus artículos destacar el potencial de aplicación de estos datos para la gestión y la planeación de la movilidad urbana y para las políticas públicas.

Para cada uno de los usuarios, se disponen de varias opciones para planear su ruta, la pregunta, es si este tiempo estimado es parecido al tiempo real.

Todo esto está estructurado con base en cartografía y datos georreferenciados que se extraen de Información de datos abiertos y de geo información que los mimos usuarios ingresan a las plataformas, pero no se consideran algunas variables que afectan la movilidad y que los demás usuarios podrían necesitar para tomar la mejor decisión para planear su viaje.

En las aplicaciones (Apps) hay que revisar cuales variables son consideradas para ajustar estos tiempos como congestión, tiempos de caminata y modo a utilizar para el viaje, entre otros. En los últimos años para los usuarios la variable que determina su planeación no es el tiempo de viaje, si no hay factores adicionales que las aplicaciones no tienen en cuenta como ocupación de los medios de transporte, paraderos y portales, operación de la infraestructura, condiciones ambientales, seguridad ciudadana entre otros.

\section{Sinestralidad vial de Bogotá}

Bogotá cuenta con un repositorio de datos de siniestralidad vial robusto que permanentemente es actualizado, a través del convenio con la Policía de Tránsito. Esta entidad registra la información de los siniestros viales a través del Informe Policial de Accidentes de Tránsito (IPAT), la cual es codificada en el Sistema de Información Geográfico de Accidentes de Tránsito (SIGAT). El proceso de registro de la información de siniestralidad vial ha tenido importantes mejoras en los últimos años, generando información sistemática y cada vez más confiable, que es analizada por la Secretaría Distrital de Movilidad realizando un reporte de manera anual.

La Secretaría Distrital de Movilidad, presenta el Anuario de datos en siniestralidad vial (SIMUR 2021). Publicación seriada que presenta un análisis comprensivo de los principales indicadores de seguridad vial, los cuales son utilizados por la entidad como herramienta de toma de decisión en política pública. Desde la Administración Distrital se entiende que la siniestralidad vial es una problemática de salud pública, que supera en número otras enfermedades como el EPOC, el cáncer de cuello uterino, la diabetes y el cáncer de mama. Así mismo, supera el número de muertes por conflicto armado, suicidio y muertes accidentales en Bogotá.

Esta Información está disponible en el sistema de Información sobre la movilidad Urbana y Regional, en el Anuario de Sinestralidad en en la cual se presentan los anuarios y las bases de datos para los años 2017-2018 y 2019.

Según la base de datos para el 2019 se presentaron 34990 siniestros reportados, en la Tabla 1 se presenta la distribución por mes y por la gravedad de siniestro.

Tabla 1: Siniestros por mes y tipo de gravedad. Fuente: Secretaría de Movilidad (2019).

\begin{tabular}{c|c|c|c|c} 
Mes & $\begin{array}{c}\text { Con } \\
\text { Heridos }\end{array}$ & $\begin{array}{c}\text { Con } \\
\text { Muertos }\end{array}$ & $\begin{array}{c}\text { Solo } \\
\text { Daños }\end{array}$ & Total \\
\hline Enero & 926 & 25 & 1540 & 2491 \\
Febrero & 999 & 38 & 1688 & 2725 \\
Marzo & 1199 & 44 & 1883 & 3126 \\
Abril & 1000 & 39 & 1785 & 2824 \\
Mayo & 1168 & 46 & 1993 & 3207 \\
Junio & 1062 & 40 & 1681 & 2783 \\
Julio & 1093 & 52 & 1826 & 2971 \\
Agosto & 1074 & 37 & 1971 & 3082 \\
Septiembre & 1048 & 42 & 1940 & 3030 \\
Octubre & 995 & 57 & 1911 & 2963 \\
Noviembre & 874 & 31 & 1860 & 2765 \\
Diciembre & 933 & 41 & 2049 & 3023 \\
Total & 12371 & 492 & 22127 & 34990
\end{tabular}


Se observa que los meses de mayo y diciembre son los meses con más accidentes con daños, meses que corresponden a meses de festivos y más viajes recreativos.

En la Tabla 2, se presenta el tipo de gravedad y el tipo de accidente, encontrando que el mayor tipo es el de choque simple, en menor medida incendio y volcamiento.

Tabla 2: Siniestros por Tipo de Choque y Tipo de Gravedad. Fuente: Secretaría de Movilidad (2019).

\begin{tabular}{|c|c|c|c|c|}
\hline $\begin{array}{l}\text { Tipo de } \\
\text { Choque }\end{array}$ & $\begin{array}{l}\text { Con } \\
\text { Heridos }\end{array}$ & $\begin{array}{c}\text { Con } \\
\text { Muertos }\end{array}$ & $\begin{array}{c}\text { Solo } \\
\text { Daños }\end{array}$ & Total \\
\hline Atropello & 3325 & 237 & 8 & 3570 \\
\hline $\begin{array}{l}\text { Caida de } \\
\text { ocupante }\end{array}$ & 852 & 7 & & 859 \\
\hline Choque & 7557 & 194 & 21976 & 29727 \\
\hline Incendio & & & 10 & 10 \\
\hline Otro & 163 & 2 & 23 & 188 \\
\hline Volcamiento & 474 & 52 & 110 & 636 \\
\hline Total & 12371 & 492 & 22127 & 34990 \\
\hline
\end{tabular}

De los 34990 siniestros, el mayor tipo es del de choque con 29727, y el segundo es atropello. De los 492 siniestros reportados con muertos, el mayor tipo corresponde a Atropello con 237 siniestros, seguido por 194 por choque y 52 por volcamiento, como se muestra en la Tabla 3.

Tabla 3: Siniestros por Localidad y Tipo de Gravedad. Fuente: Secretaría de Movilidad (2019).

\begin{tabular}{c|c|c|c|c} 
Localidad & $\begin{array}{c}\text { Con } \\
\text { Heridos }\end{array}$ & $\begin{array}{c}\text { Con } \\
\text { Muertos }\end{array}$ & $\begin{array}{c}\text { Solo } \\
\text { Daños }\end{array}$ & Total \\
\hline Antonio Nariño & 287 & 5 & 322 & 614 \\
Barrios Unidos & 505 & 11 & 1253 & 1769 \\
Bosa & 865 & 37 & 939 & 1841 \\
Candelaria & 72 & 4 & 111 & 187 \\
Chapinero & 516 & 13 & 1573 & 2102 \\
Ciudad Bolivar & 708 & 32 & 718 & 1458 \\
Engativa & 1092 & 45 & 2229 & 3366 \\
Fontibon & 762 & 38 & 2198 & 2998 \\
Kennedy & 1687 & 53 & 2195 & 3935 \\
Los Martires & 429 & 12 & 702 & 1143 \\
Puente Aranda & 911 & 41 & 1633 & 2585 \\
Rafael Uribe Uribe & 475 & 27 & 433 & 935 \\
San Cristobal & 596 & 24 & 515 & 1135 \\
Santa Fe & 421 & 20 & 638 & 1079 \\
Suba & 940 & 41 & 1893 & 2874 \\
Sumapaz & & 2 & & 2 \\
Teusaquillo & 508 & 18 & 1284 & 1810 \\
Tunjuelito & 497 & 20 & 517 & 1034 \\
Usaquen & 771 & 26 & 2653 & 3450 \\
Usme & 329 & 23 & 321 & 673 \\
Total & 12371 & 492 & 22127 & 34990 \\
& & & &
\end{tabular}

Analizando los accidentes por localización la localidad con mayor cantidad de siniestros son la localidad de Kennedy y la de Usaquen, corresponde a localidades con varios generadores de tráfico, y que en este momento tienen los corredores más congestionados de Bogotá.

Para el año 2019, según la base de datos en los 34990 siniestros están involucrados 66179 conductores, de los cuales 7401 son mujeres 57801 son hombres y según la base 1697 no registran información, aplicando un análisis de histograma clasificándolos en 10 clases. Datos que se pueden observar en la Figura 1 por rango de edades.

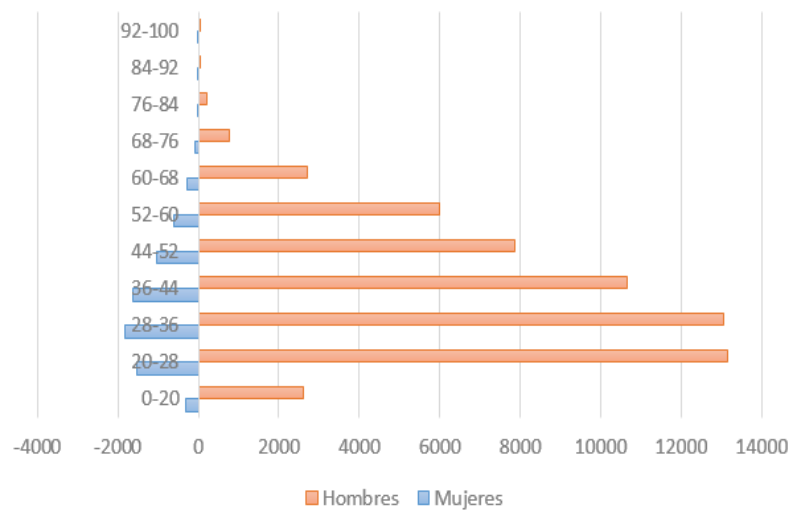

Figura 1: Clasificación de Conductores por Sexo. Fuente: Secretaría de Movilidad (2019).

\section{Metodología propuesta}

La hipótesis de partida es que las aplicaciones utilizadas, en este momento no consideran los gustos o necesidades de los usuarios o actores viales, de igual manera no consideran todas las variables para determinar el tiempo real de viaje, como son las demoras por semaforización, por espera de rutas, tiempo de espera de los buses que alimentan el sistema, congestión y demás variables.

Los objetivos de la investigación son:

- Caracterizar cada una de las aplicaciones que son utilizadas por los usuarios de transporte en la ciudad de Bogotá, para conductores, pasajeros y bici usuarios.

- Realizar encuestas a cada uno de los usuarios, que permita caracterizar las necesidades de cada uno de ellos, ya que por causas de la pandemia cambiaron los objetivos que cada usuario busca.

- Realizar comparaciones entre la planeación del tiempo de viaje con las aplicaciones y con los tiempos de viaje real con toma de datos en campo con vehículo de prueba, que permita establecer las demoras relacionadas para cada uno de los usuarios.

- Plantear un modelo que permita a los actores viales, establecer la mejor planeación de ruta de acuerdo a sus necesidades.

Para lograr estos objetivos se plantea las siguientes actividades principales:

- En primer lugar, se validarán los tiempos de recorrido planeados con las aplicaciones (Apps) vs los tiempos reales tomados en campo para cada uno de los 
usuarios, en el cual se puedan determinar las demoras y tiempos adicionales. Se contarán con apoyo de tesistas de maestría, que permita realizar estudios para cada uno de los usuarios viales.

- En segundo lugar, se realizarán encuestas a cada uno de los usuarios para conocer las prioridades o necesidades que tienen cada uno de ellos para planear sus viajes, que permita conocer estas características antes y después de la pandemia. Para conductores y pasajeros se utilizarán los medios de distribución masivo de las Universidades Distrital y la Universidad Nacional de Colombia.

- En tercer lugar, establecer un modelo para que cada uno de los actores viales pueda planear sus viajes de acuerdo a sus necesidades y que pueda contar con información para estimar el tiempo real de viaje.

\section{Aplicaciones (Apps) utilizadas}

En Colombia las principales aplicaciones (Apps) utilizadas para la planeación de rutas y navegación por los conductores son Google Maps y Waze, Google Maps es un servicio desarrollado por Google con el cual se puede encontrar la ubicación de ciudades, negocios, hoteles $O$ atracciones en Internet $O$ a través de aplicaciones en tu smartphone. Puedes considerar Google Maps como una búsqueda vertical de ubicaciones. Para la visualización se utilizan tanto datos de satélite como imágenes aéreas. Con Street View, que está integrado en Google Maps, también puedes ver carreteras y casas en determinadas regiones. La navegación y la planificación de rutas también son posibles con Google Maps (RYTE 2020)

Waze es una aplicación social de tránsito automotor en tiempo real y navegación asistida por GPS desarrollada por Waze Mobile. El 11 de junio de 2013, Google completó la adquisición de Waze, Esta aplicación proporciona direcciones en tiempo real que se ajustan sobre la marcha para tener en cuenta varios tipos de obstáculos potenciales con los aportes e informaciones dada por conductores que usan la aplicación llamados Wazers, como obras, actividades de la policía, accidentes en incluso también reporta estado de la superficie de pavimento como baches.

Según la encuesta de movilidad (Secretaria de Movilidad, 2019), la principal aplicación utilizada en Bogota es Waze, la cual tiene una utilización por estratos socioeconómicos está entre el $32 \%$ y el $58.6 \%$, según la Figura 2, esta aplicación permite compartir rutas de tránsito, dar a conocer eventualidades, como trancones o reducciones de velocidad y con esto hacer saber a otros conductores qué les puede ocurrir en la carretera que van a tomar.

Para otros conductores, principalmente en los estratos bajos utilizan Google Maps, es un servidor de aplicaciones de mapas en la web que pertenece a Alphabet Inc. Para los pasajeros de Servicio Público Individual existe una aplicación denominada Easy Taxi, ahora bajo el paraguas de Cabify. Con este tipo de aplicaciones se recibe la información de tu chófer y el número de placa. El conductor a su vez confirmará la identidad del pasajero para una mayor seguridad. De igual manera existen aplicaciones como Uber y beat que son para prestar el servicio con vehículos particulares, el cual es este momento es ilegal.
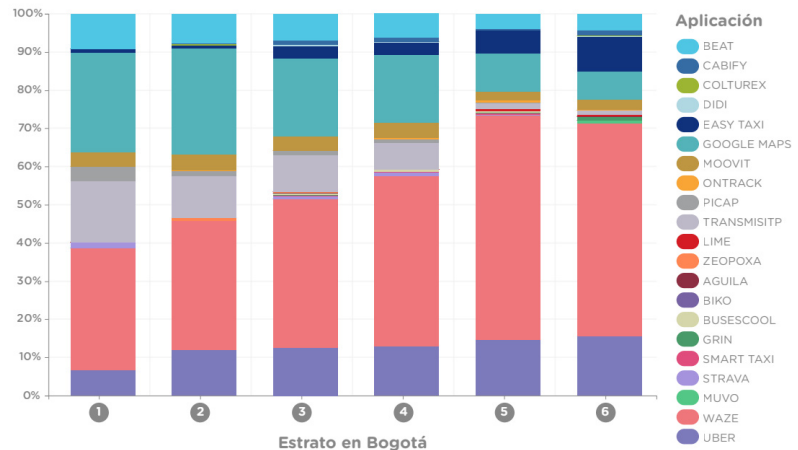

Figura 2: Distribución porcentual de viajes en los que se utilizó una aplicación móvil durante el viaje para planear o realizar el viaje. Fuente: Secretaría de Movilidad (2019).

Para los Bici usuarios, según la página de Bogotá, (https://bogota.gov.co/), se creó una nueva aplicación denominada Mapas de Bogotá Bici con la cual los usuarios pueden calcular la mejor ruta, estimar el tiempo, el trayecto a recorrer, la hora de llegada, información de interés sobre dónde parquear su bici, conocer las zonas seguras, compartir el recorrido por medio de otras aplicaciones y visualizar el estado del clima durante todo el trayecto.

Para los usuarios del transporte público está la aplicación Moovit, la cual es una app que tiene una alianza con el sistema de transporte de tipo BRT (Transmilenio) y permite elegir entre todos los servicios ofrecidos (Troncal, Alimentador, Urbano, Complementario, Especial), teniendo en cuenta las condiciones del tráfico en tiempo real generadas por informes de toda la comunidad. $Y$ la aplicación TransmiSitp la cual busca la ruta con menos paradas para moverte entre dos estaciones del sistema, además accede a la información de cada una de las rutas, incluyendo estaciones, buses, horarios, alimentadores y mapas. De igual manera presenta las rutas del sistema integrado de transporte público bogotano (sitp), urbano, complementario y especial), visualiza mapas y tablas de rutas.

\section{Comparación de aplicaciones}

Para cada uno de los usuarios, se disponen de varias opciones para planear su ruta, la pregunta, es si este tiempo estimado es parecido al tiempo real. A Grabowski (2018), se le ocurrió preguntarse cuál lo hacía mejor: Google Maps, Apple Maps o Waze (que también es de Google). Así que comparó 120 viajes hechos en San Francisco, California, donde le pidió a los tres sistemas que le calcularan una ruta y le estimaran una duración de las mismas (en promedio, de 26 minutos).

Después, comparó el tiempo estimado por cada servicio con el que finalmente le tomó recorrer esa distancia, en su estudio encontró con que Waze es el más entusiasta (el servicio que promete llegar a destino más rápido), pero también el más impreciso. Apple Maps, por el contrario, es el más precavido (calcula que llevará más tiempo el recorrido) y tiene una tasa de error relativamente alta. El que logró una mayor tasa de aciertos a la hora de calcular la duración de un trayecto 
pedido por Grabowski es Google Maps. (Grabowski 2018).

Esta misma metodología se ha planteado para Bogotá Colombia que seguirá de forma general la misma metodología, se realizará la planeación de la ruta en las dos aplicaciones más utilizadas, Waze y Google Maps, en la Figura 3 se muestra el ejemplo de planeación de la zona 113 Bavaria (Localidad de Kennedy) a la zona 108 Zona Industrial (Localidad de Puente Aranda).

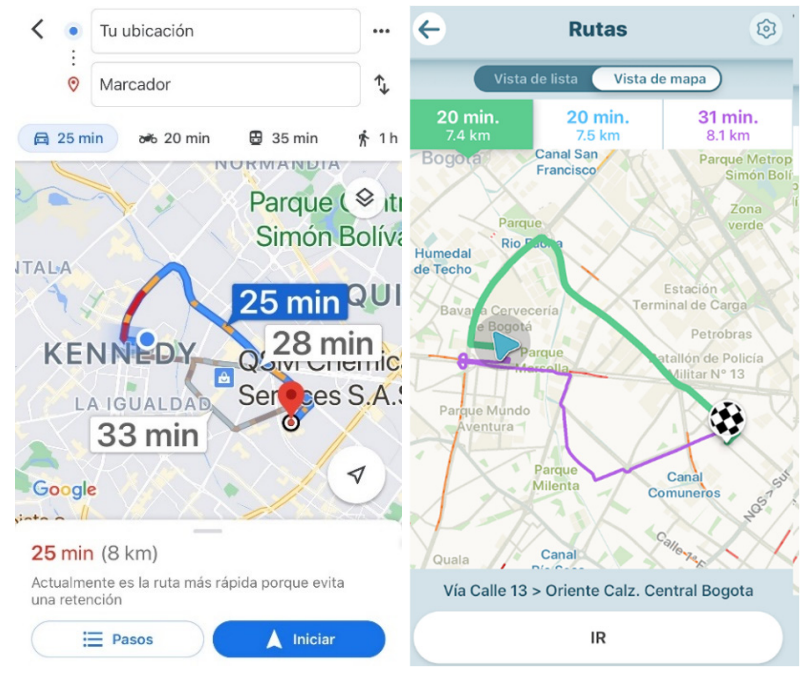

Figura 3: Planeación de Viaje en aplicaciones

Con vehículos de prueba se realizarán los recorridos para determinar el verdadero tiempo de viaje y con equipos audiovisuales adaptados al vehículo (Vargas et al. 2012), como se muestra en la Figura 4, se podrá analizar las demoras adicionales que tiene el recorrido siguiendo la metodología del vehículo en movimiento del Manual para la planeación del Tránsito de Santafé de Bogotá de la Secretaria de Movilidad de Bogotá. (Secretaría de Tránsito y Transporte 2015).

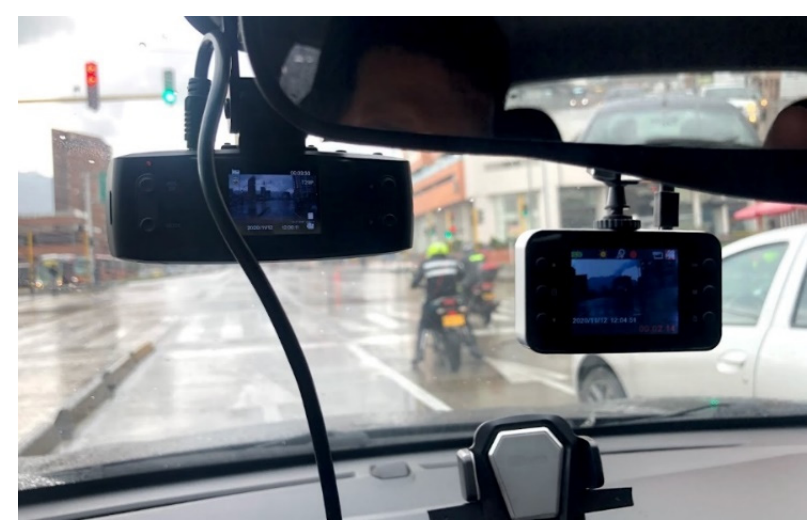

Figura 4: Vehículo de Prueba.

Con esta información se tendrá el tiempo de viaje real de viaje, y poder analizar las variaciones con el tiempo de planeación, poder establecer el tiempo de marcha, y los tiempos de demora en cada recorrido para determinar cuáles son los principales elementos que están afectando la movilidad de la ciudad, con base en los videos de las rutas (ver Figura 5).

\section{Resultados}

Debido a la pandemia el comportamiento de la movilidad es diferente, ya que, aunque en este momento en Bogotá se está activando la economía (Ramírez 2020), no se están presentando todos los viajes ya que todavía los estudiantes de Colegio y Universitarios siguen en clases virtuales, de igual manera la administración ha solicitado a los empresarios que las actividades que se puedan realizar de manera no presencial se sigan realizando apoyados con las TIC, por tal razón se han realizado algunas pruebas pero se espera tener información consolidada cuando ya se tenga la economía completamente activa y la movilidad de manera normal.

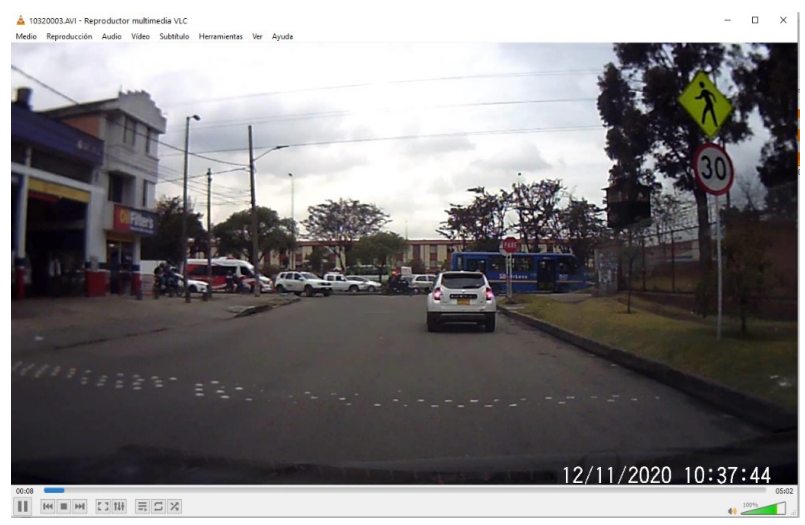

Figura 5: Vídeos de Recorrido.

De los primeros resultados obtenidos, realizados para validar la metodología, las aplicaciones presentan más o menos el mismo tiempo de viaje, presentan variaciones en la propuesta de rutas, pero la ruta principal es la misma. En la Tabla 4 se muestran los datos de recorridos.

Los tiempos de viaje reales vs los mismos tiempos de viaje planeados, tienen una variación de más o menos el $10 \%$, dependen de la distancia del viaje y de las zonas de destino, ya que los centros históricos de la ciudad presentan más demoras.

Si se relacionan los tiempos de viaje planeados frente a los tiempos reales se tiene la siguiente correlación, ver Figuras 6 y 7.

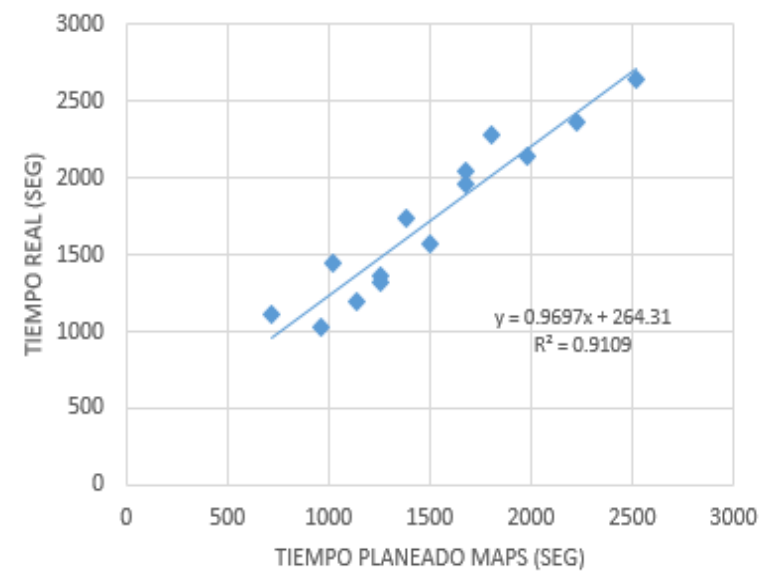

Figura 6: Comparación de tiempo real vs tiempo planeado con Waze. 
Tabla 4: Datos de Recorridos.

\begin{tabular}{l|c|c|c|c|c|c|c} 
Número & \multicolumn{9}{|c}{ Planeación } & \multicolumn{3}{c}{ Diferencia (min) } \\
& maps & waze & Incio & Final & Real & maps & waze \\
\hline 1 & $0: 33: 00$ & $0: 34: 00$ & $11: 35: 16$ & $12: 10: 53$ & $0: 35: 37$ & 2.6 & 1.6 \\
2 & $0: 37: 00$ & $0: 37: 00$ & $11: 02: 27$ & $11: 41: 48$ & $0: 39: 21$ & 2.3 & 2.3 \\
3 & $0: 23: 00$ & $0: 25: 00$ & $16: 07: 32$ & $16: 36: 29$ & $0: 28: 57$ & 6.0 & 4.0 \\
4 & $0: 21: 00$ & $0: 16: 00$ & $10: 20: 29$ & $10: 43: 16$ & $0: 22: 47$ & 1.8 & 6.8 \\
5 & $0: 42: 00$ & $0: 43: 00$ & $10: 00: 12$ & $10: 44: 19$ & $0: 44: 07$ & 2.1 & 1.1 \\
6 & $0: 25: 00$ & $0: 21: 00$ & $10: 40: 58$ & $11: 07: 09$ & $0: 26: 11$ & 1.2 & 5.2 \\
7 & $0: 30: 00$ & $0: 23: 00$ & $17: 37: 39$ & $18: 15: 36$ & $0: 37: 57$ & 8.0 & 15.0 \\
8 & $0: 21: 00$ & $0: 20: 00$ & $11: 04: 53$ & $11: 26: 54$ & $0: 22: 01$ & 1.0 & 2.0 \\
10 & $0: 28: 00$ & $0: 31: 00$ & $10: 47: 34$ & $11: 20: 13$ & $0: 32: 39$ & 4.7 & 1.7 \\
11 & $0: 28: 00$ & $0: 29: 00$ & $14: 38: 14$ & $15: 12: 16$ & $0: 34: 02$ & 6.0 & 5.0 \\
12 & $0: 16: 00$ & $0: 16: 00$ & $9: 50: 19$ & $10: 07: 20$ & $0: 17: 01$ & 1.0 & 1.0 \\
14 & $0: 12: 00$ & $0: 17: 00$ & $11: 56: 36$ & $12: 15: 04$ & $0: 18: 28$ & 6.5 & 1.5
\end{tabular}

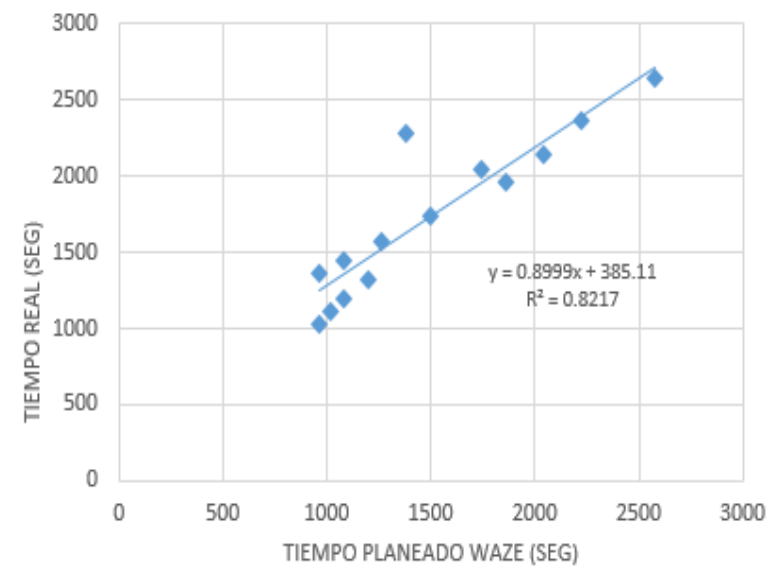

Figura 7: Comparación de tiempo real vs tiempo planeado con Waze.

Las demoras principalmente encontradas que aumentan el tiempo de viaje son: Semáforos: ya que en algunas zonas no están programados, Congestión: por diferentes causas como semáforos, accidentes viales, y vehículos estacionados, Transporte Publico: ya que no se utilizan de la mejor manera los paraderos establecidos.

Si se comparan los tiempos reales y los tiempos planeados por Maps y Waze, los tiempos reales son mayores, aunque tienen en mismo comportamiento, en la Figura 8 se presentan gráficamente estos 14 recorridos de prueba.

Los tiempos planeados, mantienen el mismo comportamiento, siempre por debajo del tiempo real, para algunos recorridos la diferencia es muy pequeña 1 a 2 minutos, y unos llegan hasta 15 minutos.

Se espera que al resetablecer las condiciones de movilidad después de la denominada nueva normalidad en Colombia, se tome la información con apoyo de los usuarios viales, como taxis, estudiantes de la universidad que utilizan el transporte publico y utilizan modos alternos como bicicletas.

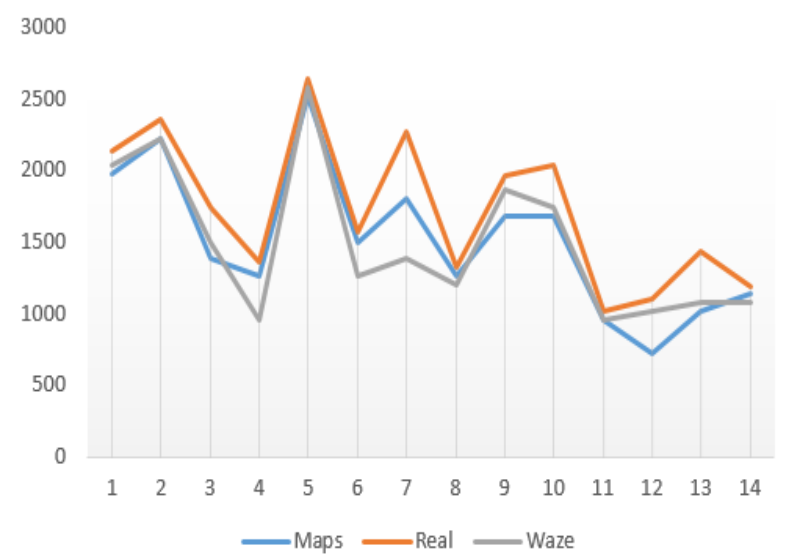

Figura 8: Comparación de tiempo real vs tiempo planeado con Waze y Maps.

\section{Conclusiones}

La utilidad de la investigación es, caracterizar las aplicaciones que son utilizadas por cada uno de los actores viales que se mueven en la ciudad de Bogotá, y determinar las demoras asociadas al tiempo de viaje, que permitirá que estos actores puedan planear de mejor manera sus viajes y desplazamientos en la ciudad.

De igual manera conocer cuáles son las necesidades que tienen cada uno de estos actores, ya que el tiempo de viaje con las condiciones actuales de la pandemia en el mundo, pueden haber cambiado y se tienen otras prioridades.

Con el modelo planteado, los usuarios viales, podrán planear mejor sus viajes de acuerdo a sus necesidades y a las condiciones reales de operación de cada medio de 
transporte, con lo que se podrá mejorar la movilidad de la ciudad, ya que los usuarios tendrán información más acertada de los tiempos de viaje a utilizar en sus viajes.

En los últimos años para los usuarios la variable que determina su planeación no es el tiempo de viaje, si no hay factores adicionales que las aplicaciones no tienen en cuenta como ocupación de los medios de transporte, paraderos y portales, operación de la infraestructura, condiciones ambientales, seguridad ciudadana entre otros.
Otro objetivo para trabajos futuros será establecer un modelo que le permita a los diferentes actores viales realizar la planeación de las rutas de acuerdo a sus necesidades y en función del tiempo real de viaje. De igual manera caracterizar las necesidades de los usuarios se determinarán por medio de encuestas en campo (Mora-Navarro et al. 2018), y por medio de mediciones de campo obtener los tiempos adicionales de viaje de cada una de las variables encontradas.

\section{References}

GRABOWSKI, A., 2018. Waze, Google Maps o Apple Maps, ¿cuál es mejor? - La Opinión (laopinion.com). Available: https://laopinion.com/2018/02/21/waze-google-maps-o-apple-maps-cual-es-mejor/ [7/24, 2021].

INRIX. 2020. Global Traffic Scorecard. INRIX Research. Available: https://inrix.com/scorecard/ [7/24, 2021].

ISER. 2010. Institute for Social-Ecological Research, Frankfurt am Main, Germany. El futuro de la movilidad en la UE Think Tank (europa.eu), Bruselas, (c) Parlamento Europeo. Available: https://www.europarl.europa.eu/thinktank/es/document.html?reference=IPOL-TRAN_NT(2010)431581 [7/24, 2021].

MORA-NAVARRO, G., FEMENIA-RIBERA, C., MARTINEZ-LLARIO, J., and ANTEQUERA-TERROSO, E., 2018. Optimising urban routes as a factor to favour sustainable school transport. Journal of Transport Geography, 72.

PARRADO, C.C., 2001. Como mejorar la Movilidad de la Ciudad. Redacción El Tiempo. Available: https://www.eltiempo.com/archivo/documento/MAM-523761 [7/24, 2021].

QUESSEP-ALCOVÉ, A. K., 2019. Problemas en movilidad, un freno para el desarrollo - De la idea a la acción | Blogs Portafolio. Available: https://blogs.portafolio.co/de-la-idea-a-la-accion/2019/07/23/problemas-movilidad-frenodesarrollo/ [7/24, 2021].

RAMÍREZ, J. M., 2020. Bogotá presentó medidas económicas para empresas después de covid-19. Revista Dinero.

RUIZ-SÁNCHEZ, T., MARS-AICART, L., and ARROYO-LÓPEZ, R., 2020. Movilidad Y Bienestar Durante EI Confinamiento Por Covid-19 En La Comunitat Valenciana. Valencia: Universitat Politécnica de Valéncia.

RYTE. 2020. ¿Qué es Google Maps? - Ryte Digital Marketing Wiki. Available: https://es.ryte.com/wiki/Google_Maps [7/24, 2021].

SABINO, A. B., REIS-MARTINS, P., and CARRANZA-INFANTE, M. (2020). Experiencias y retos del uso de datos de aplicaciones móviles para la movilidad urbana. Revista de Arquitectura (Bogotá), 22(1), pp. 82-93.

SECRETARÍA DE MOVILIDAD. 2019. Secretaría Distrital de Movilidad. Caracterización de la movilidad -Encuesta de Movilidad de Bogotá 2019. 1-23.

SECRETARÍA DE TRÁNSITO Y TRANSPORTE, 2015. Primer Encuentro Virtual de Secretarios y Autoridades de Territorio y Movilidad. Available: (https://ingenieria.bogota.unal.edu.co/es/noticias/item/427-i-encuentro-virtual-de-secretarios-yautoridades-de-territorio-y-movilidad.html [7/24, 2021].

SIMUR. 2021. Sistema Integrado de Información sobre Movilidad Urbana regional. Anuario de Sinestratidad. Available: https://www.simur.gov.co/portal-simur/datos-del-sector/documentos/anuario-de-siniestralidad/ [7/24, 2021].

VARGAS, W., RINCÓN, M. A., and GONZÁLEZ, C. J., 2012. Ingeniería de tránsito Conceptos Básicos. Colombia. 